\title{
The Seroprevalence of Dengue in a Tertiary Care Hospital
}

\author{
S. Dhivya Lakshmi, P. Nanthini Devi* and Chitralekha Saikumar \\ Department of Microbiology, Sree Balaji Medical College and hospital, Chennai, \\ Tamil Nadu, India \\ *Corresponding author
}

\begin{abstract}
A B S T R A C T
Keywords

Dengue, Aedes mosquito, Capture ELISA

Article Info

Accepted:

04 August 2018

Available Online:

10 September 2018

Dengue or break bone fever is a deadly disease caused by the Flavivirus. There are approximately 390 million dengue infections every year in the tropical and sub-tropical regions of the world. It is caused by the day biting Aedes mosquitoes which has expanded its geographical boundaries recently. Early diagnosis of Dengue is essential to prevent the complications. The study was done in a tertiary care hospital to have an insight about the seroprevalence during the dry months from March to August. The seropositivity was found to be $33.64 \%$ with the maximum number of cases in the $21-30$ years age group $(37.57 \%)$. The number of cases recorded was the highest in the month of August. $62.98 \%$ of cases were due to primary Dengue. The study highlights the importance of mosquito control measures all the year round and not just during the monsoon season.
\end{abstract}

\section{Introduction}

Dengue fever is a deadly arboviral disease caused by any of the five serotypes DENV1, DENV2, DENV 3, DENV 4 and DENV 5 which are placed in the Family Flaviviridae and genus Flavivirus (Murray et al., 2013; Mustafa et al., 2015). Infection with any one serotype provides lifelong immunity to the particular serotype but are at risk of infection to the other serotypes (Mustafa et al., 2015). Approximately 390 million dengue infections occur every year, of which 96 million manifests clinically with any severity of disease and 128 countries are at risk of infection with dengue viruses (Bhatt et al., 2013; Brady et al., 2012). The fever is caused by the day biting Aedes mosquitoes Aedes aegyptius and Aedes albopictus (Gyawali et al., 2016). Dengue can present as classic Dengue fever, Dengue hemorrhagic fever (DHF) and Dengue shock syndrome (DSS). Classic Dengue fever is characterized by sudden onset of high fever, retro orbital pain, myalgia, arthralgia and rash and occurs mostly in adults. DHF occurs mostly in children less than 15 years of age and caused by increased capillary permeability and plasma leakage leading to thrombocytopenia, bleeding and hemoconcentration and shock (termed Dengue shock syndrome) (Hadinegoro, 2012). To prevent the untoward complications of Dengue, it is imperative that early diagnosis is established. It can be done by the detection of NS1 (nonstructural protein) by RT-PCR which is not available in all hospitals and health centers. Serologic diagnosis relies on the testing of fourfold rise in antibodies by ELISA 
(Enzyme linked immunosorbent assay). Both IgM and IgG ELISA testing can be used for the differentiation of primary and secondary infections (CDC Dengue-Laboratory Guidance and Diagnostic Testing). The present study was done in a tertiary care hospital in South India and gives an insight into the seroprevalence of dengue fever and its relation to seasonal variations.

\section{Materials and Methods}

\section{Study design and period}

This is a cross sectional study done at the Central Microbiology laboratory of a tertiary care hospital between March 2017 and August 2017 for a period of six months. The blood collection was performed under strict aseptic precautions. Sera were separated after centrifuging and were subjected to ELISA testing of NS1 antigen, $\operatorname{IgM}$ and $\operatorname{IgG}$ antibodies by the JMitra kit.

\section{Inclusion criteria}

All patients in any age group presenting with fever, headache, rash, retroorbital pain, myalgia and other symptoms suspicious of Dengue fever from various outpatient, inpatient departments and emergency services.

\section{Exclusion criteria}

Fever confirmed due to non-infectious causes or other causes such as Leptospira, Hepatitis and Typhoid.

\section{Blood collection and processing}

About $5 \mathrm{ml}$ of blood was collected under strict aseptic precautions. Sera were separated after centrifuging at $1000 \mathrm{rpm}$ for 2 minutes and were subjected to ELISA testing of NS1 antigen, IgM and IgG antibodies by the JMitra kit (Microwell ELISA Test).

\section{Precautions}

Specimens should be free of microbial contamination and may be stored at $2-8^{0} \mathrm{C}$ for one week, or frozen at $-20^{0} \mathrm{C}$ or lower. Repeated freezing and thawing should be avoided. Hemolyzed and hyperlipemic samples may give erroneous results.

\section{Procedure}

DENGUE NS1 Ag MICROLISA is a screening test designed for the qualitative detection of Dengue NS1 antigen in serum or plasma. The kit detects all four dengue subtypes; DEN1, DEN2, DEN3 and DEN4 of Dengue Virus. It is a solid phase enzyme linked immunosorbent assay (ELISA) based on the "Direct Sandwich" principle. The samples are added in the microwells which are coated with Anti-dengue NS1antibodies followed by addition of enzyme conjugate (monoclonal anti-dengue NS1antibodies linked to Horseradish Peroxidase enzyme (HRPO)). A sandwich complex is formed, where the dengue NS1 from patient's serum is trapped between the antibody and antibody HRPO conjugate. The unbound conjugate is washed off with wash buffer. Upon addition of the substrate buffer and chromogen, a blue color develops. The intensity of development is proportional to the concentration of dengue NS1 antigen in sample. A stop solution is added to limit the enzyme-substrate reaction and a yellow colour develops which is read at $450 \mathrm{~nm}$ spectrophotometrically.

The sensitivity of Dengue NS1 Ag Microlisa kit was found to be $98.28 \%$.

\section{IgM/IgG CAPTURE ELISA}

Dengue $\operatorname{IgM} / \mathrm{IgG}$ Microlisa test is an enzyme immunoassay based on "CAPTURE ELISA". Anti-human IgM/IgG antibodies are coated onto microtiter wells. Serum samples and 
controls are added to the microtiter wells and incubated. Antibodies to Dengue, will bind to the anti- human $\operatorname{IgM} / \mathrm{IgG}$ antibodies adsorbed onto the surface of the wells followed by washing to remove unbound material.

Horseradish peroxidase (HRPO) conjugated Dengue antigen (DEN-4) is added to each well. This dengue antigen conjugate will bind to dengue specific $\operatorname{IgM} / \operatorname{IgG}$ antibodies which is complex with anti-human IgM antibodies.

Finally substrate solution containing chromogen and hydrogen peroxide is added to the wells. A blue colour develops according to the amount of dengue antibodies present in the sample. The reaction is stopped by a stop solution. The enzyme substrate reaction is ready by EIA reader for absorbance at a wavelength of $450 \mathrm{~nm}$. The sensitivity was found to be $99.13 \%$ and specificity was found to be $99.84 \%$.

\section{Results and Discussion}

The total number of patients whose serum was sent to the Central Microbiology laboratory for the six months study period was 538 out of which $276(51.3 \%)$ were males and 262 (48.7\%) were females (Fig. 1).

The seropositivity for Dengue NS1, IgM, IgG by ELISA was 181 (33.64\%) out of which 92 $(50.83 \%)$ were males and $89(49.17 \%)$ were females (Fig. 2).

Dengue is an important and life threatening arboviral infection in tropical countries with an estimated 390 million infection and 96 million symptomatic infections occurring annually (Bhatt et al., 2013). The early diagnosis of Dengue is of great importance to arrest the progression of Dengue related complications. The total number of patients whose serum was sent to the Central Microbiology laboratory on suspicion of
Dengue, for the six months study period was 538 out of which 276 (51.3\%) were males and $262(48.7 \%)$ were females.

The seropositivity of Dengue in our study was $33.64 \%$ out of which $92(50.83 \%)$ were males and $89(49.17 \%)$ were females. Gopal et al., (2016) reported a seroprevalence of $50 \%$, Gupta et al., reported a positivity of $29.09 \%$, Kalaivani et al., (2016) reported a 62\% seroprevalence which is very higher compared to our study.

The table 1 shows increased prevalence in the male gender in many studies, but a study by Kalaivani et al., (2016) reports equal prevalence. Our study also supports this finding showing an increased prevalence towards the male gender. This is due to the fact that males are involved in more outdoor activities compared to females.

Madan et al., (2018) reported $26.47 \%$ in the 21-30 years age group followed by $21.56 \%$ in 11-20 years. Mahesh Kumar et al., (2015) reported high prevalence in the 10-20 years age group (31.58\%). Sujatha et al., (2016) recorded maximum prevalence in the age group 21- 30 years $(55.09 \%)$ followed by 11 20 years (27.54\%). Bhat et al., (2013) reported an increased prevalence in adults $>15$ years (Mishra et al., 2016) (Fig. 3).

Patankar et al., (2014) reported 51\% in the age group of 18-35 years followed the age group 5-17 years age group (20\%). But our study showed high prevalence of seropositivity in the 21-30 years age group-37.57\% followed by $18.58 \%$ in the $11-20$ years age group.

The high incidence of seropositivity in the 1130 years shows that the children and young adults were exposed to mosquito bites due to their habits of involving in brisk outdoor activities. 
Fig.1 Shows distribution of male and female among the study group

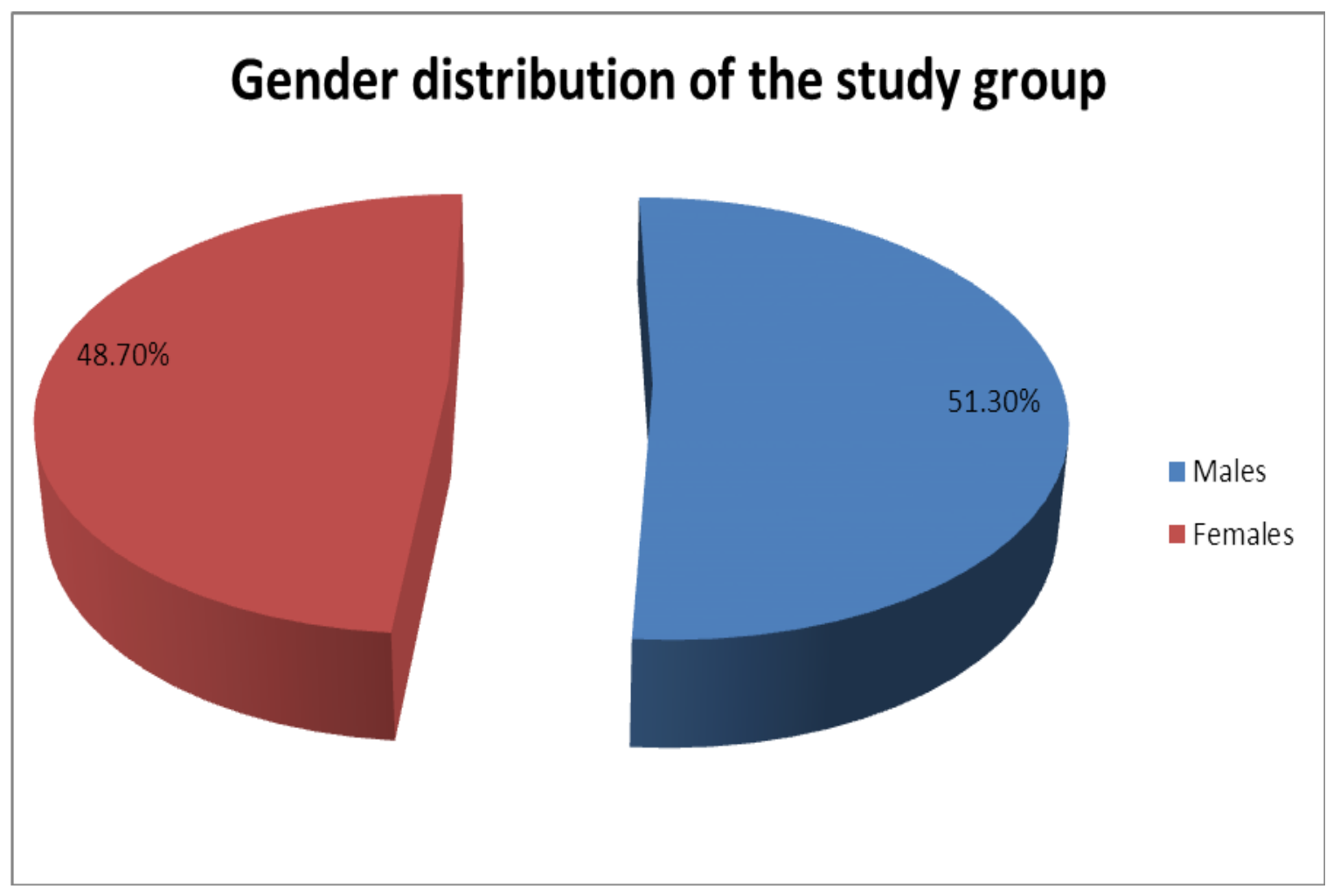

Fig.2 Shows distribution of male and female among dengue positive cases

\section{Gender distribution of Dengue positive patients}

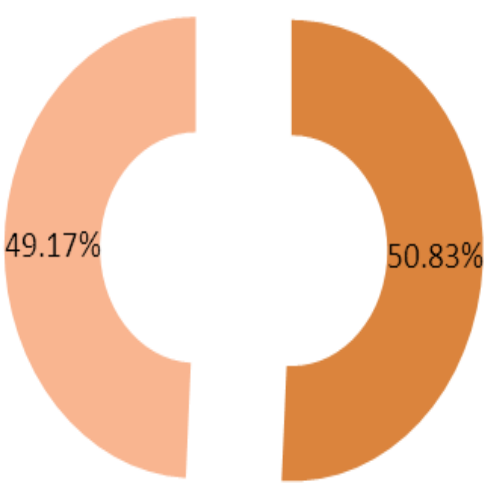

Males

Females 
Fig.3 Age wise distribution of dengue positive patients

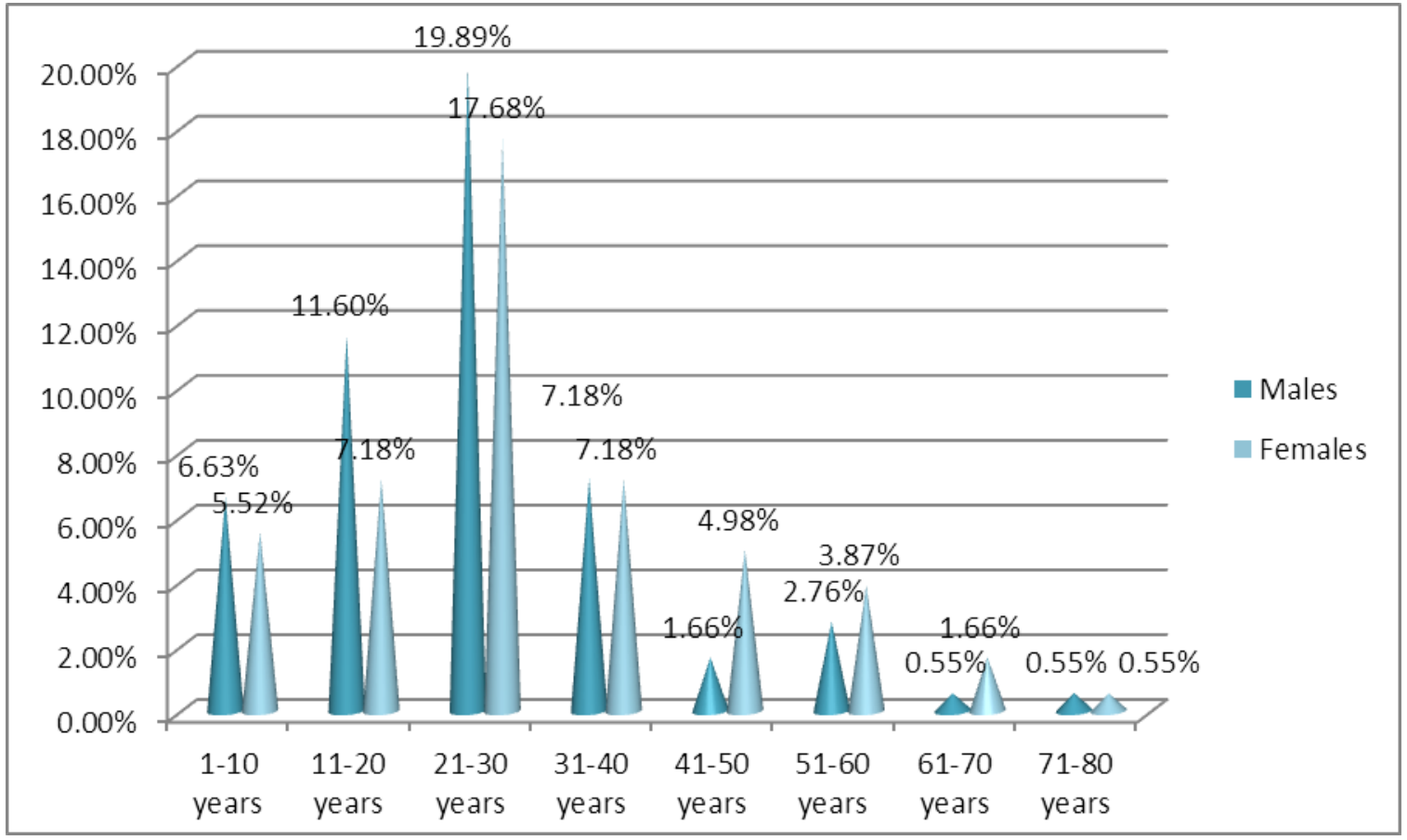

Fig.4 A graph that shows the prevalence of dengue in relation to months of the year

\section{PREVALENCE OF DENGUE IN RELATION TO MONTHS OF THE YEAR}

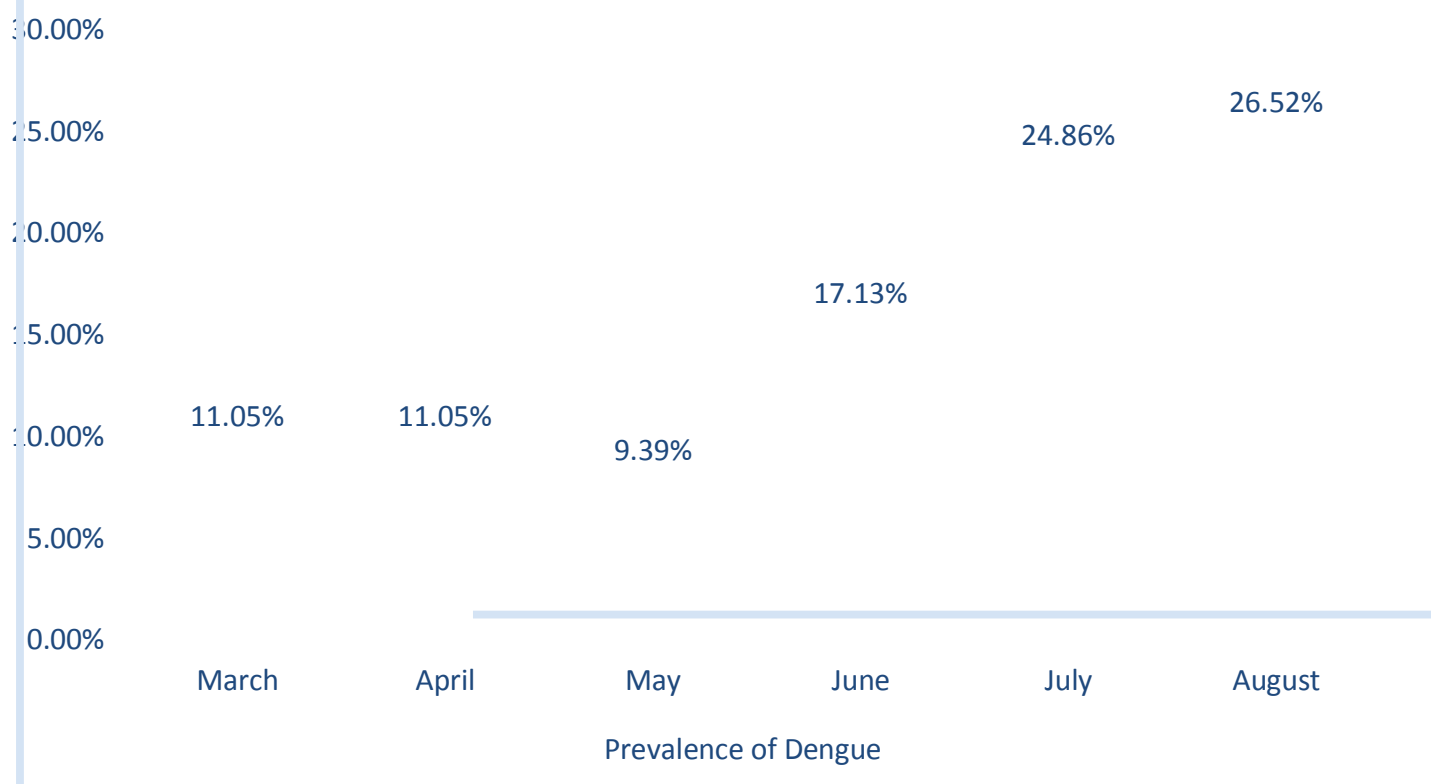


Table.1 Dengue Elisa NS1, IgM, IgG positivity with respect to gender

\begin{tabular}{|l|l|l|l|l|}
\hline \multirow{2}{*}{$\begin{array}{l}\text { Positivity of } \\
\text { antigen/antibody }\end{array}$} & Males & $\%$ & Females \\
\hline NS1 & 39 & $21.55 \%$ & 52 & $\%$ \\
\hline IgM & 11 & $6.08 \%$ & 3 & $1.66 \%$ \\
\hline IgG & 16 & $8.84 \%$ & 17 & $9.39 \%$ \\
\hline IgM \& IgG & 8 & $4.42 \%$ & 7 & $3.87 \%$ \\
\hline NS1 \& IgM & 7 & $3.87 \%$ & 2 & $1.1 \%$ \\
\hline NS1 \& IgG & 5 & $2.76 \%$ & 4 & $2.21 \%$ \\
\hline NS1, IgM \& IgG & 6 & $3.31 \%$ & 4 & $2.21 \%$ \\
\hline
\end{tabular}

Table.2 Types of infection in dengue

\begin{tabular}{|l|}
\hline Type of infection \\
\hline Primary infection \\
\hline Late primary /Early secondary infection \\
\hline Secondary infection \\
\hline Past infection \\
\hline
\end{tabular}

\begin{tabular}{|l|l|}
\hline Number & Percent \\
\hline 114 & $62.98 \%$ \\
\hline 15 & $8.29 \%$ \\
\hline 19 & $10.5 \%$ \\
\hline 33 & $18.23 \%$ \\
\hline
\end{tabular}

Table.3 Shows prevalence of dengue in various studies

\begin{tabular}{|l|l|l|}
\hline S.NO & PREVALENCE & REFERENCE \\
\hline 1. & $50 \%$ & Gopal et al., (2016) \\
\hline 2. & $29.09 \%$ & Gupta et al., \\
\hline 3. & $62 \%$ & Kalaivani et al., (2016) \\
\hline 4. & $20 \%$ & Goswami et al.,(2018) \\
\hline 5. & $53.2 \%$ & Srinivas Rao et al., (2013) \\
\hline 6. & $21.65 \%$ & Madan et al., (2018) \\
\hline 7. & $3.55 \%$ & Mahesh kumar et al., (2015) \\
\hline 8. & $37.38 \%$ & Sujatha et al., (2016) \\
\hline 9. & $33.64 \%$ & Present study \\
\hline
\end{tabular}

Table.4

\begin{tabular}{|l|l|l|l|}
\hline S. No & Males & Females & Reference \\
\hline 1. & $50 \%$ & $50 \%$ & Kalaivani et al., (2016) \\
\hline 2. & $77.3 \%$ & $23.7 \%$ & Mishra et al., (2016) \\
\hline 3. & $61.76 \%$ & $38.23 \%$ & Madan et al., (2018) \\
\hline 4. & $62.63 \%$ & $37.37 \%$ & Mahesh kumar et al., (2015) \\
\hline 5. & $61 \%$ & $39 \%$ & Sujatha et al., (2016) \\
\hline 6. & $65 \%$ & $35 \%$ & Patankar et al., (2014) \\
\hline 7. & $50.83 \%$ & $48.17 \%$ & Present study \\
\hline
\end{tabular}


The highest number of cases was recorded in November followed by October and December according to Mahesh Kumar et al., (2015). Sujatha et al., (2016) recorded maximum cases in October and November. Bhat et al., (2013) also reported increased prevalence of dengue in the season after rainfall (KSB et al., 2014). Patankar et al., (2014) reported increased positivity in October. But Nissi Mathew et al., (2017) reported increased prevalence in August which is different from other studies.

Though these studies highlight the fact that the prevalence of dengue increases in the after monsoon, our study shows that there is an increased prevalence even in the dry seasons (March-11.05\%, April-11.05\%, May-9.39\%, June-17.13\%, July-24.86\%, August-26.52\%), which shows the year round transmission of Dengue. So there is a dire need to take precautionary measures to control mosquitoes throughout the year and not just in the season after monsoon (Fig. 4).

The antibody response to dengue viral infection varies. When there is a primary infection with dengue, IgM antibody is the first antibody to rise by 3-5 days after the illness, peaks by the $10^{\text {th }}$ day and declines by 2-3 months. IgG antibodies rise by the end of the first week of illess, rises slowly and remains detectable in serum for many months or even for life. In contrast when there is secondary infection due to dengue virus or any other flavivirus infection or vaccination, IgG antibodies rise rapidly and the level of IgM antibodies is lower compared to IgG antibody (Dengue: Guidelines for Diagnosis, Treatment, Prevention and Control, 2009) (Table 2).

In a study by Gopal et al., (2016) 26\% was primary infection and $30 \%$ was due to secondary infection. Srinivasa Rao et al., (2013) reported $17.7 \%$ of primary infection,
$28.05 \%$ of past infection, $54.25 \%$ of patients in the convalescent phase. Madan et al., (2018) reported $87.25 \%$ of primary infection, $5.88 \%$ patients of late primary or early secondary infection, $6.86 \%$ cases, suggesting secondary or past infection. Kalaivani et al., (2016) recorded $43.57 \%$ of primary dengue and $56.43 \%$ of secondary dengue. But in the present study, $62.98 \%$ of cases were due to primary infection, $10.5 \%$ due to secondary infection, $8.29 \%$ due to early secondary/late primary infection and $18.23 \%$ due to past infection (Table 3 and 4).

The chief drawback of the study was that paired sera samples were not used for testing which will differentiate the primary and secondary infection clearly. Confirmatory testing also was also not done by RT-PCR to determine the serotype.

Dengue has become a threat even in the current era. There are a lot of challenges in the control of Dengue. The vector Aedes mosquito has invaded new territories due to climatic changes and there is lack of suitable diagnostic facilities. The serologic testing of Dengue is not reliable as there is the problem of cross reactivity. Though Dengue is a notifiable disease, the number of cases reported is underestimated due to the lack of proper diagnostic tools.

There are no licensed vaccines for Dengue. Even when a vaccine is available it should cover all the serotypes of Dengue virus.

Vector control is the only measure to control the spread of Dengue but there is the problem of insecticide resistance. Personal protective measures to avoid mosquito bites should be undertaken to break the transmission of Dengue.

Conflict of interest: None Source of funding: None 


\section{References}

Ajay Gopal K, Kalaivani V, Anandan H. Prevalence of Dengue Fever and Comparative Analysis of $\operatorname{IgM}$ and $\operatorname{IgG}$ Antibodies in Dengue Fever in Thoothukudi-Southern Coastal City, Tamil Nadu. Ann.Int. Med. Den. Res. 2016; 2(6):MB04-MB07

Alera MT, Srikiatkhachorn A, Velasco JM, Tac-An IA, Lago CB, Clapham HE, et al., (2016) Incidence of Dengue Virus Infection in Adults and Children in a Prospective Longitudinal Cohort in the Philippines. PLoSNegl Trop Dis 10(2): e0004337.

Bhatt S, Gething PW, Brady OJ, Messina JP, Farlow AW, Moyes CL et al., The global distribution and burden of dengue. Nature. 2013; 496: 504-7. doi:10.1038/nature 12060.

Brady OJ, Gething PW, Bhatt S, Messina JP, Brownstein JS, Hoen AG et al., Refining the global spatial limits of dengue virus transmission by evidencebased consensus. PLoSNegl Trop Dis. 2012; 6: e1760. doi:10.1371/ journal.pntd.0001760.

CDC Dengue-Laboratory Guidance and Diagnostic Testing

Dengue: Guidelines for Diagnosis, Treatment, Prevention and Control: New Edition. Geneva: World Health Organization; 2009. 4, Laboratory Diagnosis and Diagnostic Tests.

Goswami L, Chowdhury R, Rasul ES. Seroprevalence of dengue infection in a tertiary care hospital in Assam. Int $\mathbf{J}$ Med and Dent Sci 2018; 7(1): 15821585 .

Gupta, B.P., S K Mishra, K D Manandhar, R Malla, C S Tamarakar, P P Raut, S K Sah, S Pokhrel, $\mathrm{R}$ Rauniyar, A. Bajaracharya. Seroprevalence of Dengue Virus Infection in Nepal. Int $\mathbf{J}$ Appl Sci Biotechnol, Vol. 1(4): 224-227
Gyawali N, Bradbury RS, Taylor-Robinson AW. The epidemiology of dengue infection: Harnessing past experience and current knowledge to support implementation of future control strategies. J Vector Borne Dis 53, December 2016, pp. 293-304

Hadinegoro SRS. The revised WHO dengue case classification: does the system need to be modified? Paediatrics and International Child Health. 2012; 32(s1): 33-38. doi:10.1179/ $2046904712 Z .00000000052$.

Kalaivani V, Ajay Gopal K, Srinivasakannan, Anandan H. Prevalence of Dengue Fever in Kanyakumari District: A Cross-sectional Study. Int J Sci Stud 2016; 4(6): 158-160.

KSB, Sastry AS, Senthamarai S, Sivasankari S. Seroprevalence of dengue viral infection in patients attending to a tertiary care hospital in Kanchipuram, Tamil Nadu, India. Int J Res Health Sci (Internet). 2014 Jul 31; 2(3): 818-22.

Kumar M, Sharma R, Parihar G, Sharma M. Seroprevalence of Dengue in Central Rajasthan: A Study at a Tertiary Care Hospital. Int.J.Curr.Microbiol.App.Sci (2015) 4(9): 933-940

Madan SP, Bhatawadekar S, Lahiri K. Clinico-demographic profile and seroprevalence of dengue at a tertiary care hospital-study from Maharashtra. Int J Health Sci Res. 2018; 8(1):43-48.

Mathew N, Rajahamsan J, Sahira H, Rani B, Ramani Bai J.T. Study on Prevalence of Dengue Fever in a Tertiary Care Hospital, South Kerala. JMSCR. January 2017; 5(1): 15435-15440.

Mishra S, Ramanathan R, Agarwalla SK, "Clinical Profile of Dengue Fever in Children: A Study from Southern Odisha, India," Scientifica, 2016; 2016: 1-6. Article ID 6391594.

Murray NEA, Quam MB, Wilder-Smith A. Epidemiology of dengue: past, present 
and future prospects. Clinical Epidemiology. 2013; 5: 299-309. doi:10.2147/CLEP.S34440.

Mustafa MS, Rasotgi V, Jain S, Gupta V. Discovery of fifth serotype of dengue virus (DENV-5): A new public health dilemma in dengue control. Medical Journal, Armed Forces India. 2015; 71(1):67-70. doi:10.1016/j.mjafi.2014.09.011.

Patankar MC, Patel BV, Gandhi VP, Shah PD, Vegad MM. Seroprevalence of dengue in Gujarat, Western India: A study at a tertiary care hospital. Int $\mathbf{J}$ Med Sci Public Health 2014; 3: 16-18. Srinivas Rao MS, Pavani K, Dass M, Khareem MA, Vinayaraj EV. Seroprevalence of Dengue in a tertiary care hospital in Andhra Pradesh, south India. Int J Res Med Sci 2013; 1: 44850

Sujatha R, Pal N, Prachi S. Seroprevalence Of Dengue Fever In A Tertiary Care Center At Kanpur. Rama Univ. J. Med Sci 2016; 2(1): 15-19

\section{How to cite this article:}

Dhivya Lakshmi, S., P. Nanthini Devi and Chitralekha Saikumar. 2018. The Seroprevalence of Dengue in a Tertiary Care Hospital. Int.J.Curr.Microbiol.App.Sci. 7(09): 43-51.

doi: https://doi.org/10.20546/ijcmas.2018.709.006 